\title{
Stability analysis of systems with nested saturation and backlash in the loop via nonstandard anti-windup compensation
}

\author{
Sophie Tarbouriech, Isabelle Queinnec, Christophe Prieur
}

\begin{abstract}
This paper deals with control systems subject to backlash and saturation in the loop. One of the goals is to characterize, or at least to approximate, the attractor of such nonlinear dynamical systems. Then, the regional or global asymptotic stability of the closed loop with respect to this attractor is handled. An anti-windup inspired loops is added aiming at improving the quality of the attractor approximation in which converge the closed-loop trajectories and of the basin of attraction of such an attractor. Numerically tractable algorithms with feasibility guarantee are provided, as soon as the linear closed-loop system, obtained by neglecting the backlash and saturation effects, is asymptotically stable. The interest of the results is drawn through an illustrative example.
\end{abstract}

\section{INTRODUCTION}

Physical systems are often subject to constraints or limitations. Indeed, the constraints affecting the control systems can be due to physical, technological, safety or communication reasons. Also, technological progresses in material and communication sciences for key technologies such as mechanical or piezo devices are used more and more in space, aeronautical, biotechnology and health areas. Then, associated safety requirements motivate extensive research on analysis and control design of complex systems subject to several constraints across all engineering disciplines [9]. In general, control engineers prefer to work under the assumption of linearity, in particular due to the fact that the associated mathematics is well developed and rather easy to apply. Such an assumption of linearity is sometimes unrealistic and can lead to erroneous results, even is impossible to be done depending on the nonlinearity considered. Actually, the increasing requirements in terms of operational reliability and performance ask to work beyond the linear behavior of the system and therefore to be able to take explicitly into account the constraints affecting the system. Hence, in the paper we want to study control systems subject to both saturation and backlash operators as studied in particular in [12], [18] for the case of saturations, or [6], [7] for the case of backlash operators.

Despite the interest to take into account these joint nonlinearities, few results can be found in the literature to deal with stability analysis or control design purpose. In [3], the authors study the presence of saturation and backlash in series at the input of the system, and invert the backlash

S. Tarbouriech and I. Queinnec are with LAAS-CNRS, Université de Toulouse, CNRS, Toulouse, France tarbourdaas.fr, queinnec@laas. fr

$\begin{array}{cccc}\text { C. Prieur is with Univ. Grenoble Alpes, } & \text { CNRS, } \\ \text { Grenoble-INP, GIPSA-lab, F-38000, Grenoble, } & \text { France } \\ \text { christophe.prieurdgipsa-lab.fr } & \end{array}$ in order to cancel its undesirable effects. That leads to a system with a new saturation with a level depending on the magnitude constraint of the previous saturation and on the parameters of the backlash. In [13], the closed-loop system is a dynamical one with nested backlash and saturation operators issued from a static output feedback. The contribution of the current paper can be viewed as an extension of [13] in the sense that we do not consider a static output feedback but a dynamic output feedback and we search for designing an anti-windup term to mitigate the combined effects of saturation and backlash. Inspired both by [5] and [15], the technique does not intend to invert the backlash element but uses it directly to build the anti-windup loop. Stability analysis conditions are derived from the use of Lyapunov theory arguments and generalized sector conditions using properties of the saturation and backlash operators, in a regional (local) or global context. The regional result (see Theorem 3.1 below) consists in defining a compact set (inner set), which is a finite-time attractor, for all admissible initial conditions belonging to another compact set (outer set). Then, the second contribution of the paper (see Theorem 3.2) deals with the global case. In this case, the outer set becomes all the state space, provided that the open-loop system is not strictly exponentially unstable. Furthermore, let us specify that the backlash operator considered is a component-wise model as in [6], [10], [16], [1], [8].

The paper is organized as follows. In Section II, the system under consideration and the problem we intend to solve are described. Section III presents the theoretical conditions to design the anti-windup loop and discuss their feasibility. Computational issues are discussed and illustrated in Section IV. Some concluding remarks point out the future developments end the paper.

Notation. For two vectors $x, y$ of $\mathbb{R}^{n}$, the notation $x \succeq y$ means that $x_{(i)}-y_{(i)} \geq 0, \forall i=1, \ldots, n .1$ and $\mathbf{0}$ denote the identity matrix and the null matrix of appropriate dimensions, respectively. $x \in \mathbb{R}_{+}^{n}$ means that $x \succeq 0$. The Euclidian norm is denoted $\|\cdot\| \cdot A^{\prime}$ and $\operatorname{trace}(A)$ denote the transpose and the trace of $A$, respectively. $\operatorname{He}\{A\}=A+A^{\prime}$. $\operatorname{diag}(A ; B)$ denotes the diagonal matrix which diagonal blocks are formed by square matrices $A$ and $B$. For two symmetric matrices, $A$ and $B, A>B$ means that $A-B$ is positive definite. In partitioned symmetric matrices, the symbol $\star$ stands for symmetric blocks. $\lambda_{\max }(A)$ (respectively, $\lambda_{\min }(A)$ ) denotes the maximal (respectively, minimal) eigenvalue of the matrix A. 


\section{PROBLEM FORMULATION}

The class of systems under consideration is described by:

$$
\begin{aligned}
& \dot{x}_{p}=A_{p} x_{p}+B_{p} u_{p} \\
& y_{p}=C_{p} x_{p}
\end{aligned}
$$

where $x_{p} \in \mathbb{R}^{n_{p}}$ is the state, $u_{p} \in \mathbb{R}^{n_{p}}$ is the input of the plant and $y_{p} \in \mathbb{R}^{m}$ is the measured output of the plant. $A_{p}$, $B_{p}$ and $C_{p}$ are matrices of appropriate dimensions. The pairs $\left(A_{p}, B_{p}\right)$ and $\left(A_{p}, C_{p}\right)$ are supposed to be controllable and observable.

Throughout the paper, the connection between the plant and the controller through the output of the controller $y_{c} \in$ $\mathbb{R}^{m}$ is defined by

$$
u_{p}=\operatorname{sat}\left(\Phi\left[y_{c}\right]\right)
$$

and depicted in Figure 1.

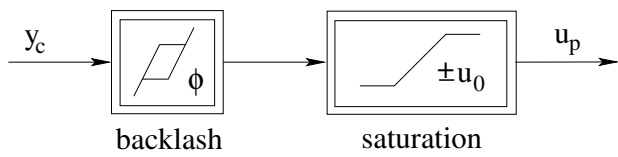

Fig. 1. Closed-loop system with backlash and saturation in the actuator

In (2) and Figure 1, sat denotes the classical vector-valued saturation function defined as $\forall i=1, \ldots, m:(\operatorname{sat}(z))_{(i)}=$ $\operatorname{sat}\left(z_{(i)}\right)=\operatorname{sign}\left(z_{(i)}\right) \min \left(u_{0(i)},\left|z_{(i)}\right|\right)$ with $u_{0(i)}>0$ the $i$ th level of the saturation. $\Phi$ is a component-wise backlash operator (see, for example, [6], [10], [16], [1]). We denote by $\mathcal{C}_{p w}^{1}\left([0,+\infty) ; \mathbb{R}^{m}\right)$ the set of continuous, piecewise differentiable functions $f:[0,+\infty) \rightarrow \mathbb{R}^{m}$, that is the set of continuous functions $f$ being, for some unbounded sequence $\left(t_{j}\right)_{j=0}^{\infty}$ in $[0,+\infty)$ with $t_{0}=0$, continuously differentiable on $\left(t_{j-1}, t_{j}\right)$ for all $j \in \mathbb{N}$. Given the vector $\rho$ in $\mathbb{R}_{+}^{m}$ and $L=\operatorname{diag}\left(\ell_{(i)}\right)$, with $\ell_{(i)}>0, i=1, \ldots, m$, the operator $\Phi$ is defined as follows, for all $f \in \mathcal{C}_{p w}^{1}\left([0,+\infty) ; \mathbb{R}^{m}\right)$, for all $j \in \mathbb{N}$, for all $t \in\left(t_{j-1}, t_{j}\right)$ and for all $i \in\{1, \ldots, m\}$ :

$$
\overbrace{\Phi[f]}(t))_{(i)}=\left\{\begin{array}{c}
\ell_{(i)} \dot{f}_{(i)}(t) \text { if } \dot{f}_{(i)}(t) \geq 0 \\
\text { and }(\Phi[f](t))_{(i)}=\ell_{(i)}\left(f_{(i)}(t)-\rho_{(i)}\right) \\
\ell_{(i)} \dot{f}_{(i)}(t) \text { if } \dot{f}_{(i)}(t) \leq 0 \\
\quad \begin{array}{l}
\text { and }(\Phi[f](t))_{(i)}=\ell_{(i)}\left(f_{(i)}(t)+\rho_{(i)}\right) \\
0 \text { otherwise }
\end{array}
\end{array}\right.
$$

where $0=t_{0}<t_{1}<\ldots$ is a partition of $[0,+\infty)$ such that $f$ is continuously differentiable on each of the intervals $\left(t_{j-1}, t_{j}\right), j \in \mathbb{N}$. Thus, $\Phi$ is a time-invariant nonlinearity with slope restriction, as in [11]. Note however that it is a memory-based operator, since to compute it, we need to have information about the past values of its input (this is not the case in [11]).

The plant is supposed to be controlled by the following output dynamical controller

$$
\begin{aligned}
& \dot{x}_{c}=A_{c} x_{c}+B_{c} y_{p}+v_{c} \\
& y_{c}=C_{c} x_{c}+D_{c} y_{p}
\end{aligned}
$$

where $x_{c} \in \mathbb{R}^{n_{c}}$ is the state, $y_{p} \in \mathbb{R}^{n_{p}}$ is the output of the plant, $y_{c} \in \mathbb{R}^{m}$ is the output of the controller and $v_{c} \in \mathbb{R}^{n_{c}}$ is an input signal available to perform a suitable correction (anti-windup action) for mitigating the undesired effects of saturation and backlash. Controller (4) with $v_{c}=\mathbf{0}$ has been designed through classical techniques, disregarding the effects of both the control saturation and the presence of the backlash nonlinearity.

Remark 2.1: By construction, the linear connection plantcontroller is supposed to be stable. In other words, the controller (4) (with $v_{c}=0$ ) stabilizes the plant (1) through the linear interconnection $u_{p}=L y_{c}$ (which corresponds to take $\operatorname{sat}\left(\Phi\left[y_{c}\right]\right)=\Phi\left[y_{c}\right]=L y_{c}$ in (2)) and therefore the matrix:

$$
A_{0}=\left[\begin{array}{cc}
A_{p}+B_{p} L D_{c} C_{p} & B_{p} L C_{c} \\
B_{c} C_{p} & A_{c}
\end{array}\right]
$$

is Hurwitz.

Nevertheless, due to the presence of both backlash and saturation in the input the real interconnection between the plant (1) and the controller (4) is issued from (2). The presence of the backlash operator $\Phi$ may induce the existence of multiple equilibrium points or a limit cycle around the origin. Furthermore, in a neighborhood of the origin, system (1) operates in open loop. In parallel, the presence of the saturation asks for characterizing suitable regions of the state space in which the stability of the closed-loop saturated system can be ensured [12].

In order to mitigate the effects of control saturation on the performance as well as on the stability of the closedloop system, an anti-windup loop may be added to the precomputed controller through the signal $v_{c}$. The principle of the considered anti-windup loop consists in picking the difference between the output of the nonlinear actuator $\left(\operatorname{sat}\left(\Phi\left[y_{c}\right]\right)\right)$ and the output of the linearized one $\left(L y_{c}\right)$ for building $v_{c}$. Thus, by selecting $v_{c}=E_{c}\left(\operatorname{sat}\left(\Phi\left[y_{c}\right]\right)-L y_{c}\right)$, with $E_{c} \in \mathbb{R}^{n_{c} \times m}$, the closed-loop system issued from (1) and (4) reads:

$$
\begin{aligned}
& \dot{x}_{p}=A_{p} x_{p}+B_{p} \operatorname{sat}\left(\Phi\left[y_{c}\right]\right) \\
& y_{p}=C_{p} x_{p} \\
& \dot{x}_{c}=A_{c} x_{c}+B_{c} y_{p}+E_{c}\left(\operatorname{sat}\left(\Phi\left[y_{c}\right]\right)-L y_{c}\right) \\
& y_{c}=C_{c} x_{c}+D_{c} y_{p}
\end{aligned}
$$

The proposed approach aims at characterizing two sets such that the closed-loop trajectories initialized in the outer set are converging to the inner set and remain confined in it. It is important to emphasize that the proposed technique does not require the open-loop system to be stable. Nevertheless, depending on the open-loop stability, the global stability context is also carried out. In this case, the outer set corresponds to the whole state space.

According to (3), one gets $\Phi\left[y_{c}\right](t) \in I_{\Phi}$ with

$$
I_{\Phi}=\left\{\Phi\left[y_{c}\right] \in \mathbb{R}^{m} ; L\left(y_{c}+\rho\right) \succeq \Phi\left[y_{c}\right] \succeq L\left(y_{c}-\rho\right)\right\}
$$

By definition, for any initial condition in $I_{\Phi}$, the solution $\Phi\left[y_{c}\right]$ remains confined in $I_{\Phi}, \forall t \geq 0$, which means that the nonlinearity $\Phi$ is active [7], [2]. Furthermore, we are 
concerned with the asymptotic behavior of the state $x=$ $\left[\begin{array}{ll}x_{p}^{\prime} & x_{c}^{\prime}\end{array}\right]^{\prime} \in \mathbb{R}^{n}, n=n_{p}+n_{c}$ but not of the operator $\Phi$. Therefore, we want to study the stability properties of the following attractor:

$$
\mathcal{A}=\mathcal{S}_{0} \subseteq \mathbb{R}^{n}
$$

Then the problems we intend to solve can be summarized as follows in regional and global contexts.

Problem 2.1 (Regional Case): Characterize the regions $\mathcal{S}_{1}$ and $\mathcal{S}_{0}$ of the state space and design the anti-windup gain $E_{c}$ such that the closed-loop trajectories of the system (6) remain confined in $\mathcal{S}_{1}$ and converge to the set $\mathcal{S}_{0}$, when initialized as in (7).

Problem 2.2 (Global Case): Assume that $A_{p}$ is Hurwitz. Characterize the region $\mathcal{S}_{0}$ of the state space, containing the origin, and design the gain $E_{c}$ such that system (6) is globally asymptotically stable with respect to $\mathcal{S}_{0}$, when initialized as in (7). In other words, $\mathcal{S}_{0}$ is a global asymptotic attractor for the closed-loop dynamics (6), for any initial value of $\Phi$ in $I_{\Phi}$.

\section{MAIN RESUlTS}

\section{A. Preliminary results}

For conciseness, throughout the paper, we denote $\dot{\Phi}$ instead of $\overbrace{\Phi\left[y_{c}\right]}$, and $\Phi$ instead of $\Phi\left[y_{c}\right]$. Let us define the nonlinearities $\varphi_{1}$ and $\Psi_{1}$, which are derived from (2)

$$
\begin{aligned}
& \varphi_{1}=\operatorname{sat}\left(\Phi\left[y_{c}\right]\right)-\Phi\left[y_{c}\right] \\
& \Psi_{1}=\Phi\left[y_{c}\right]-L y_{c}
\end{aligned}
$$

Hence, by defining the augmented state $x=\left[\begin{array}{cc}x_{p}^{\prime} & x_{c}^{\prime}\end{array}\right]^{\prime} \in$ $\mathbb{R}^{n}, n=n_{p}+n_{c}$, the closed-loop system reads:

$$
\begin{aligned}
& \dot{x}=A_{0} x+\left(B+R E_{c}\right)\left(\varphi_{1}+\Psi_{1}\right) \\
& y_{c}=K x
\end{aligned}
$$

with $A_{0}$ defined in (5) and

$$
K=\left[\begin{array}{ll}
D_{c} C_{p} & C_{c}
\end{array}\right] ; B=\left[\begin{array}{c}
B_{p} \\
\mathbf{0}
\end{array}\right] ; R=\left[\begin{array}{l}
\mathbf{0} \\
\mathbf{1}
\end{array}\right]
$$

\section{B. Regional stability}

The following result provides a solution to Problem 2.1, exploiting some results of [15].

Theorem 3.1: If there exist a symmetric positive definite matrix $W \in \mathbb{R}^{n \times n}$, three diagonal positive definite $S_{2} \in$ $\mathbb{R}^{q \times q}, T_{3} \in \mathbb{R}^{q \times q}$ and $S \in \mathbb{R}^{m \times m}$, two matrices $Z \in \mathbb{R}^{m \times n}$ and $E_{c} \in \mathbb{R}^{n_{c} \times m}$, two positive scalars $\eta, \tau$ satisfying the following conditions

$$
\begin{aligned}
& M_{1}<\mathbf{0} \\
& \rho^{\prime} L T_{3} L \rho-\tau \leq 0 \\
&\left(\begin{array}{cc}
W & Z_{(i)}^{\prime} \\
\star & \eta u_{0(i)}^{2}
\end{array}\right) \geq \mathbf{0}, i=1, \ldots, m \\
& \eta<1
\end{aligned}
$$

with $M_{1}$ defined in (14) (see at the top of the next page), then, for any initial admissible conditions $\left(x(0), \Psi_{1}(0)\right)$, with $x(0) \in \mathcal{S}_{1}$, the resulting trajectories of the closed-loop system (12) converge to the set $\mathcal{S}_{0}$, where the sets $\mathcal{S}_{1}$ and $\mathcal{S}_{0}$ are defined as follows:

$$
\begin{aligned}
& \mathcal{S}_{1}=\left\{x \in \mathbb{R}^{n} ; x^{\prime} W^{-1} x \leq \eta^{-1}\right\} \\
& \mathcal{S}_{0}=\left\{x \in \mathbb{R}^{n} ; x^{\prime} W^{-1} x \leq 1\right\}
\end{aligned}
$$

Proof: Consider a quadratic Lyapunov function candidate $V$ defined by $V(x)=x^{\prime} P x, P=P^{\prime}>\mathbf{0}$, for all $x$ in $\mathbb{R}^{n}$. Regarding the nonlinearity $\varphi_{1}$, the proof relies on the application of the generalized sector condition [12] stating that for any diagonal positive define matrix $S^{-1}$, one satisfies: $\left.-2 \varphi_{1}^{\prime} S^{-1}\left(\operatorname{sat}\left(\Phi\left[y_{c}\right]\right)\right)+G x\right) \geq 0$ for any $x \in S\left(G, u_{0}\right)=\left\{x \in \mathbb{R}^{n} ;-u_{0(i)} \leq G_{(i)} x \leq u_{0(i)}, i=\right.$ $1, \ldots, m\}$, with $G=Z W^{-1}=Z P$. Furthermore, the satisfaction of relation (16) ensures that the set $\mathcal{S}_{1}$, defined in (18) is included in the region $S\left(G, u_{0}\right)$ in which the sector condition on $\varphi_{1}$ is satisfied. Hence, in $\mathcal{S}_{1}$, one gets $\dot{V}(x) \leq \dot{V}(x)-2 \varphi_{1}^{\prime} S^{-1}\left(\operatorname{sat}\left(\Phi\left[y_{c}\right]\right)+G x\right)$. Furthermore, the satisfaction of relation (17) ensures that $\mathcal{S}_{1}$ contains $\mathcal{S}_{0}$.

We want to verify that there exists a class $\mathcal{K}$ function $\alpha$ such that $\dot{V}(x) \leq-\alpha(V(x))$, for all $x$ such that $x^{\prime} P x \geq$ 1 and $x^{\prime} P x \leq \eta^{-1}$ (i.e. for any $x \in \mathcal{S}_{1} \backslash \mathcal{S}_{0}$ ), and for all nonlinearities $\Psi_{1}$ satisfying Lemma 1 in [14].

By using the S-procedure as in [15], it is sufficient to check that $\mathcal{L}<0$, where

$$
\begin{aligned}
\mathcal{L}= & \dot{V}(x)-\tau\left(1-x^{\prime} P x\right)-\Psi_{1}^{\prime} T_{3} \Psi_{1}+\rho^{\prime} L T_{3} L \rho \\
& -2\left(\dot{\Psi}_{1}+L K \dot{x}\right)^{\prime} N_{2} \dot{\Psi}_{1} \\
& -2 \varphi_{1}^{\prime} S^{-1}\left(\operatorname{sat}\left(\Phi\left[y_{c}\right]\right)+G x\right)
\end{aligned}
$$

with $\tau$ a positive scalar and $T_{3}$ a positive diagonal matrix. Noting that $\dot{V}(x)=x^{\prime}\left(A_{0}^{\prime} P+P A_{0}\right) x+2 x^{\prime} P(B+$ $\left.R E_{c}\right)\left(\varphi_{1}+\Psi_{1}\right)$, it follows that $\mathcal{L}=\mathcal{L}_{0}+\rho^{\prime} L T_{3} L \rho-\tau$ with

$$
\mathcal{L}_{0}=\left(\begin{array}{c}
P x \\
\Psi_{1} \\
S_{2}^{-1} \dot{\Psi}_{1} \\
S^{-1} \varphi_{1}
\end{array}\right)^{\prime} M_{1}\left(\begin{array}{c}
P x \\
\Psi_{1} \\
S_{2}^{-1} \dot{\Psi}_{1} \\
S^{-1} \varphi_{1}
\end{array}\right)
$$

and $M_{1}$ defined in (14), recalling that $W=P^{-1}$. The satisfaction of relations (13) and (15) implies both $\mathcal{L}_{0}<$ 0 and $\rho^{\prime} L T_{3} L \rho-\tau \leq 0$, and then $\mathcal{L}<0$, for all $\left(x, \varphi_{1}, \Psi_{1}, \dot{\Psi}_{1}\right) \neq \mathbf{0}$.

Therefore, the satisfaction of relations (13)-(17) ensures that there exists $\varepsilon>0$, such that $\mathcal{L} \leq$ $-\varepsilon\left\|\left(x^{\prime} \varphi_{1}^{\prime} \Psi_{1}^{\prime} \dot{\Psi}_{1}^{\prime}\right)^{\prime}\right\|^{2} \leq-\varepsilon x^{\prime} x$. Hence, since by definition one gets $\dot{V}(x) \leq \dot{V}(x)-\tau_{1}\left(1-x^{\prime} P x\right) \leq \mathcal{L}$, one can also verify

$$
\dot{V}(x) \leq-\varepsilon x^{\prime} x, \forall x \text { such that } \eta^{-1} \geq x^{\prime} P x \geq 1
$$

Consider now a solution to (12) starting from any admissible initial condition at $t_{0}$ such that $\eta^{-1} \geq x\left(t_{0}\right)^{\prime} P x\left(t_{0}\right) \geq 1$. According to (21), there exists a time $T \geq t_{0}+\left(x\left(t_{0}\right)^{\prime} P x\left(t_{0}\right)-\right.$ 1) $\lambda_{\max }(P) / \epsilon$ such that $x(t) \in \mathcal{S}_{1}, \forall t \geq T$. Furthermore, $\mathcal{S}_{1}$ is an invariant set for the trajectories of system (12). 


$$
M_{1}=\left(\begin{array}{cccc}
H e\left\{A_{0} W\right\}+\tau W & B+R E_{c} & -W A_{0}^{\prime} K^{\prime} L & B S+R E_{c} S-W K^{\prime} L-Z^{\prime} \\
\star & -T_{3} & -\left(B+R E_{c}\right)^{\prime} K^{\prime} L & -\mathbf{1} \\
\star & \star & -2 S_{2} & -L K\left(B+R E_{c}\right) S \\
\star & \star & \star & -2 S
\end{array}\right)
$$

Hence, $\mathcal{S}_{0}$ is an attractor for the closed-loop trajectories. That concludes the proof of Theorem 3.1.

\section{Global stability}

Assume that the open-loop matrix $A_{p}$ is Hurwitz. Then, the following result follows.

Theorem 3.2: If there exist a symmetric positive definite matrix $W \in \mathbb{R}^{n \times n}$, three diagonal positive definite $S_{2} \in$ $\mathbb{R}^{q \times q}, T_{3} \in \mathbb{R}^{q \times q}$ and $S \in \mathbb{R}^{m \times m}$, a matrix $E_{c} \in \mathbb{R}^{n_{c} \times m}$, a positive scalar $\tau$ satisfying the following conditions

$$
\begin{gathered}
M_{2}<\mathbf{0} \\
\rho^{\prime} L T_{3} L \rho-\tau \leq 0
\end{gathered}
$$

with $M_{2}$ defined in (23) (see at the top of the next page), then, for any initial admissible conditions $\left(x(0), \Psi_{1}(0)\right)$, the resulting trajectories of the closed-loop system (12) globally converge to the set $\mathcal{S}_{0}$, with $\mathcal{S}_{0}$ defined as in (19).

Proof: The proof relies on the application of the generalized sector condition in the global case, which corresponds to take $G=\mathbf{0}$, or equivalently $Z=\mathbf{0}$ [12]. Then one satisfies: $\left.-2 \varphi_{1}^{\prime} S^{-1} \operatorname{sat}\left(\Phi\left[y_{c}\right]\right)\right) \geq 0$ for any $x \in \mathbb{R}^{n}$. The rest of the proof readily follows that one of Theorem 3.1.

\section{Feasibility}

Let us discuss about the feasibility of conditions of Theorem 3.1 or Theorem 3.2. By defining

$$
M_{0}=\left[\begin{array}{ccc}
H e\left\{A_{0} W\right\}+\tau W & \star & \star \\
B^{\prime} & -T_{3} & \star \\
-L K A_{0} W & -L K B & -2 S_{2}
\end{array}\right]
$$

and inspired by [15] and [4], we can state the following proprosition.

Proposition 3.1: Theorem 3.1 enjoys the following properties:

1) Given $E_{c}=\mathbf{0}$, condition (13) is feasible if matrix $A_{0}$ is Hurwitz.

2) There always exists $E_{c}$ non null such that condition (13) holds, if matrix $A_{0}$ is Hurwitz.

Proof: In [15], it has been shown that $M_{0}<0$ is feasible if matrix $A_{0}$ is Hurwitz (that is there exist $\tau, W, T_{3}$ and $S_{2}$ such that $M_{0}<\mathbf{0}$ ). Observe that matrix $M_{1}$ when $E_{c}=\mathbf{0}$, denoted $M_{1}(\mathbf{0})$, reads:

$$
M_{1}(\mathbf{0})=\left[\begin{array}{c}
M_{0} \\
\star
\end{array}\left[\begin{array}{c}
B S-W K^{\prime} L-Z^{\prime} \\
-\mathbf{1} \\
-L K B S \\
-2 S
\end{array}\right]\right]
$$

By inspection, it appears that if $M_{0}$ is feasible (which corresponds to the existence of $\tau, W, T_{3}$ and $S_{2}$ ), one can always find values for matrices $Z$ and $S$ such that $M_{1}(\mathbf{0})$ is feasible.

Consider now the case where $E_{c}$ is non null. Condition (14) can then be written as

$$
M_{1}=M_{1}(\mathbf{0})+H e\left\{\left[\begin{array}{c}
R \\
\mathbf{0} \\
-L K R \\
\mathbf{0}
\end{array}\right] E_{c}\left[\begin{array}{llll}
\mathbf{0} & \mathbf{1} & \mathbf{0} & S
\end{array}\right]\right\}
$$

Hence, if $M_{1}(\mathbf{0})<\mathbf{0}$ is feasible, one can always find some value for $E_{c}$ such that $M_{1}<\mathbf{0}$ is feasible with $M_{1}$ defined as in (27).

The same reasoning regarding the feasibility of condition (22) could be derived.

\section{E. Computational issues}

Regarding the analysis problem ( $E_{c}$ given), the only nonlinearity which appears in the conditions is the product $\tau W$, due to the use of the S-procedure. Such a nonlinearity is not much an issue to handle, and a grid search on $\tau$ may be simply used to find a feasible solution. Moreover, the value of $\tau$ has not too much influence on the size of the computed sets $\mathcal{S}_{0}$ (and $\mathcal{S}_{1}$ in the regional case).

Regarding the anti-windup design problem, another nonlinearity occurs in conditions (13) and (22), associated to the product between $E_{c}$ and $S$. Conditions may be slightly modified to remove this nonlinearity, thanks to the following trick: left- and right-multiply (13) by $\operatorname{diag}(\mathbf{1} ; S ; \mathbf{1} ; \mathbf{1})$ then use the perfect square property allowing to write that $-S T_{3} S \leq-2 S+T_{3}^{-1}$. Then $M_{1}$ becomes:

$\bar{M}_{1}=\left(\begin{array}{cccc}\bullet & \left(B+R E_{c}\right) S & \bullet & \bullet \\ \star & -2 S+T_{3}^{-1} & -S^{\prime}\left(B+R E_{c}\right)^{\prime} K^{\prime} L & -S \\ \star & \star & -\bullet & \bullet \\ \star & \star & \star & \bullet\end{array}\right)$

with - referring to terms unchanged in (14). By denoting $E_{c} S=Y$, equation (28) becomes linear in $W, S_{2}, S_{3}=$ $T_{3}^{-1}, S, Z$ and $Y$. conditions (15) is also modified as follows:

$$
\left[\begin{array}{cc}
-\tau & \rho^{\prime} L \\
L \rho & -T_{3}^{-1}
\end{array}\right] \leq 0
$$

Same manipulations may be applied on $M_{2}$ to build a suitable matrix $\bar{M}_{2}$, allowing to linearize the conditions of Theorem 3.2 ( $\tau$ being given).

Remark 3.1: Another option may be to solve the design problem with a given value for $S$ (typically the solution to the analysis problem). A D-K iteration procedure could even be applied at that point, but does not improve much the solution in practice. 


$$
M_{2}=\left(\begin{array}{cccc}
H e\left\{A_{0} W\right\}+\tau W & B+R E_{c} & -W A_{0}^{\prime} K^{\prime} L & B S+R E_{c} S-W K^{\prime} L \\
\star & -T_{3} & -\left(B+R E_{c}\right)^{\prime} K^{\prime} L & -\mathbf{1} \\
\star & \star & -2 S_{2} & -L K\left(B+R E_{c}\right) S \\
\star & \star & \star & -2 S
\end{array}\right)
$$

Theorems 3.1 and 3.2 may then be associated to optimization procedures regarding the size of the set $\mathcal{S}_{0}$ (to be minimized), and, for the regional case, of the set $\mathcal{S}_{1}$ (to be maximized). One can then consider:

- Regional case

$\min \eta$

subject to (13), (15), (16), (17) - analysis problem

or

subject to $\bar{M}_{1}<0$, (29), (16), (17) - design problem

- Global case

$\min \operatorname{trace}(W)$

subject to (22), (24) - analysis problem

or

subject to $\bar{M}_{2}<0$, (29) - design problem

\section{ILLUSTRATIVE EXAMPLE}

Consider the following open-loop unstable example to illustrate the local conditions stated in Section III-B with:

$$
\begin{aligned}
A_{p} & =\left[\begin{array}{ccc}
0.5 & 0 & -1 \\
1 & -1 & 0 \\
0 & 0 & 0
\end{array}\right], \quad B_{p}=\left[\begin{array}{ll}
1 & 0 \\
0 & 0 \\
0 & 1
\end{array}\right] \\
C_{p} & =\left[\begin{array}{ccc}
1 & 0 & 0 \\
0 & 0 & 1
\end{array}\right]
\end{aligned}
$$

with associated dynamic controller defined as:

$$
\begin{array}{rlrl}
A_{c} & =\left[\begin{array}{ccc}
-1.45 & 0 & 0 \\
-1 & -10 & 0 \\
4.7 & 0 & -10
\end{array}\right], & B_{c}=\left[\begin{array}{cc}
3 & -1 \\
1 & 0 \\
-1 & 10
\end{array}\right] \\
C_{c}=\left[\begin{array}{ccc}
-1 & -10 & 0 \\
3.7 & 0 & -2.7
\end{array}\right], & D_{c}=\left[\begin{array}{cc}
0 & 0 \\
0 & 0
\end{array}\right]
\end{array}
$$

An actuator involving a saturation with $u_{0}=\left[\begin{array}{ll}0.5 & 0.5\end{array}\right]^{\prime}$ and a backlash element given by $(\rho, \quad \mathrm{L})=$ $\left(\left[\begin{array}{ll}0.15 & 0.15\end{array}\right]^{\prime}, \mathbf{1}\right)$ interconnects the plant and its controller.

For $\tau=0.5$, the solution to the regional analysis problem gives:

$$
\eta=0.7719, \operatorname{vol}\left(\mathcal{S}_{0}\right)=3.7538, \operatorname{vol}\left(\mathcal{S}_{1}\right)=8.1634
$$

with $\operatorname{vol}\left(\mathcal{S}_{0}\right)=\sqrt{\operatorname{det}(W)}$ and $\operatorname{vol}\left(\mathcal{S}_{1}\right)=\sqrt{\operatorname{det}(W) / \eta}$.

Similarly, for $\tau=0.1$, the solution to the regional antiwindup design problem gives:

$$
E_{c}=\left[\begin{array}{cc}
-0.6342 & 0.1158 \\
0.7978 & -0.2303 \\
-1.4219 & 2.4875
\end{array}\right]
$$

$\eta_{a w}=0.0376, \operatorname{vol}\left(\mathcal{S}_{0 a w}\right)=6.9633, \operatorname{vol}\left(\mathcal{S}_{1 a w}\right)=1.3066 e 5$

One can check that the inner set (mainly related to the backlash effect) is reduced and the outer set (mainly related to the saturation effect) is enlarged.

Let us now consider the initial condition $x_{p}(0)=$ $\left[\begin{array}{lll}0.8 & -0.5 & 0.85\end{array}\right]^{\prime}, x_{c}(0)=\mathbf{0}$. This initial condition belongs to the external set $\mathcal{S}_{1 a w}$ but not to $\mathcal{S}_{1} . \Phi\left[y_{c}(0)\right]$ is initialized in the middle of the set $I_{\Phi}$ in order to be active. Simulations are plotted in Figures 2-5 to illustrate the influence of the anti-windup gain on the closed-loop response of the system. The plant state response without anti-windup (upper plot) and with anti-windup (lower plot) is given in Figure 2. The plant input is given in Figure 3 both in the case without anti-windup (upper plot) and with anti-windup (lower plot). The actuator response $\left(u_{p}\right.$ versus $\left.y_{c}\right)$ is also plotted in Figure 4 for the first component of the input and in Figure 5 for the second component, considering both cases without anti-windup (left plot) and with anti-windup (right plot). One can check on the figures that the anti-windup effect initially compensates for the saturation effect, then later reduces the backlash effect on the input/output behavior.
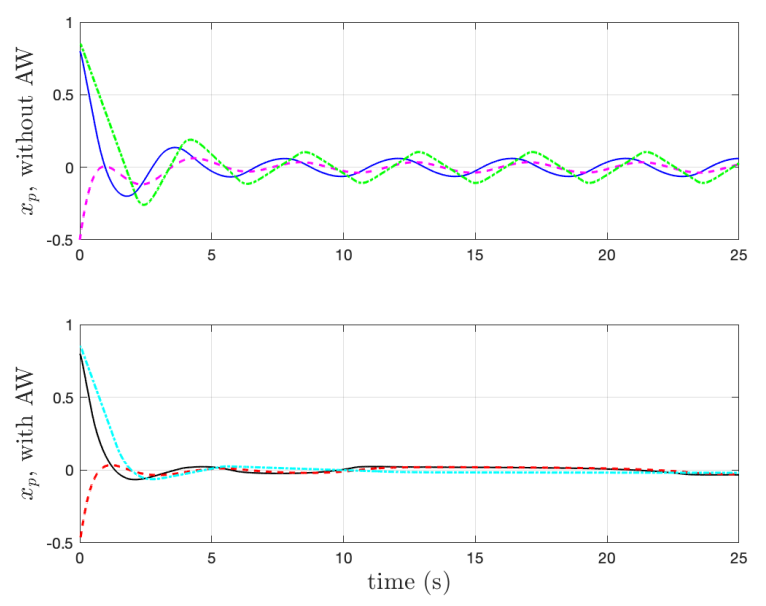

Fig. 2. Time evolution of the plant state $x_{p}$. Upper plot: case without anti-windup action. Lower plot: case with anti-windup action.

\section{CONCLUSion}

A class of nonlinear systems has been considered in this paper. The model results from the interaction of a linear plant with a linear controller and two nested nonlinear operators (saturation and backlash). The main contribution of this work is to approximate the attractor of such nonlinear dynamical systems, and to derive conditions for the design of the anti-windup loop. Both the regional and global asymptotic stability properties of the closed-loop system have been studied. Numerical simulations allow to illustrate the numerical 

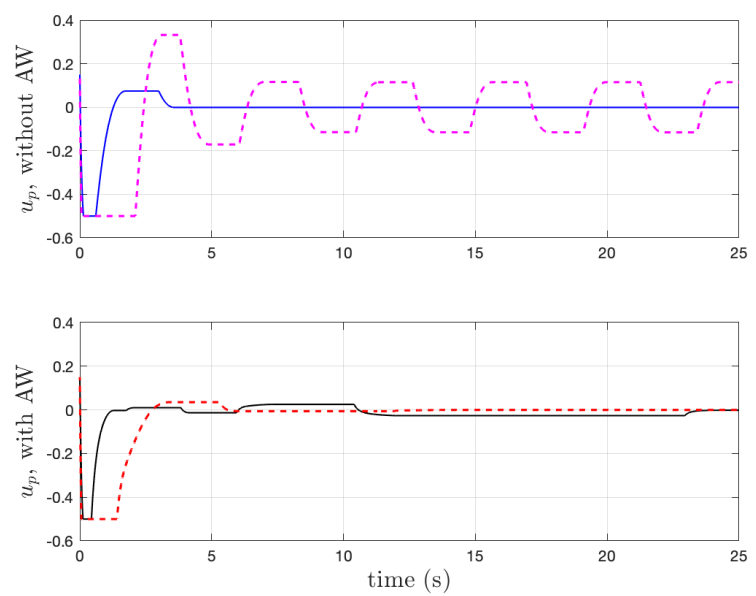

Fig. 3. Time evolution of the plant input $u_{p}$. Upper plot: case without anti-windup action. Lower plot: case with anti-windup action.
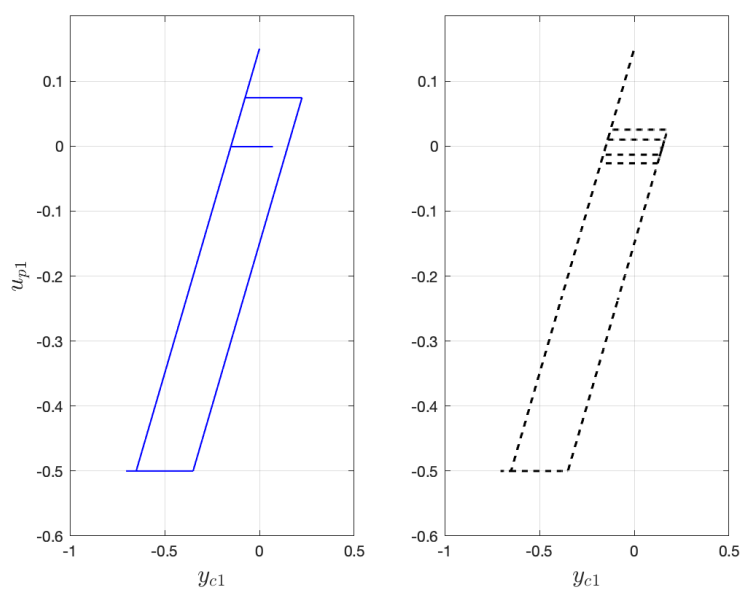

Fig. 4. Backlash characteristics $u_{p}$ versus $y_{c}$ (first component). Left plot: case without anti-windup action. Right plot: case with anti-windup action.

tractability of the synthesis conditions, and the interest of adding anti-windup inspired loops.

This work opens the door for future research lines. It could be interesting to derive conditions for the observer design problem in the case where the backlash appears in the measured output of the system. This problem may be seen as the dual problem of the problem studied in this paper. For this problem, maybe some techniques for saturated systems (as studied in particular in [17]) could be adapted and combined with the design of anti-windup loops.

\section{REFERENCES}

[1] M. Brokate and J. Sprekels. Hysteresis and phase transitions, volume 121 of Applied Mathematical Sciences. Springer-Verlag, New York, 1996.

[2] M. L. Corradini and G. Orlando. Robust stabilization of nonlinear uncertain plants with backlash or dead zone in the actuator. IEEE Transactions on Control Systems Technology, 10(1):158 - 166, 2002.
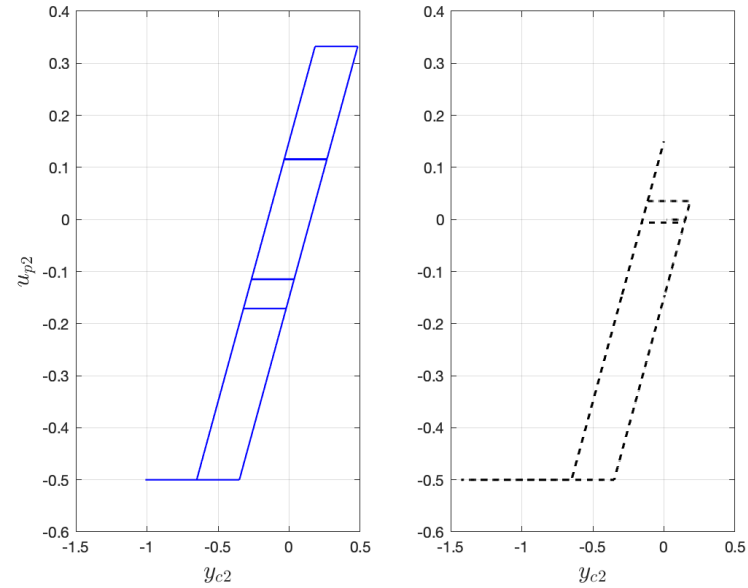

Fig. 5. Backlash characteristics $u_{p}$ versus $y_{c}$ (second component). Left plot: case without anti-windup action. Right plot: case with anti-windup action.

[3] M del Carmen Rodriguez and W.P. Heath. Controller structure for plants with combined stauration and deadzone/backlash. In International Conference on Control Application (CCA), pages 1394-1399, Dubrovnik, Croatia, October 2012.

[4] F. Ferrante, F. Gouaisbaut, and S. Tarbouriech. On sensor quantization in linear control systems: Krasovskii solutions meet semidefinite programming. IMA Journal of Mathematical Control and Information, to appear.

[5] J. M. Gomes da Silva Jr and M. C. Turner. Static anti-windup for systems with sector-bounded nonlinearities. In American Control Conference (ACC), pages 1531-1536, Montreal, Canada, June 2012.

[6] J. W. Macki, P. Nistri, and P. Zecca. Mathematical models for hysteresis. SIAM Review, 35(1):94-123, 1993.

[7] M. Nordin, X. Ma, and P.O. Gutman. Controlling mechanical systems with backlash: a survey. Automatica, 38:1633-1649, 2002.

[8] R. Ouyang and B. Jayawardhana. Absolute stability analysis of linear systems with duhem hysteresis operator. Automatica, 50:1860-1866, 2014.

[9] D. Papageorgiou, M. Blanke, H.H. Niemann, and J.H. Richter. Backlash estimation for industrial drive-train systems. In IFAC PapersOnLine, volume 50-1, pages 3281-3286, 2017.

[10] T. Paré, A. Hassibi, and J. How. A KYP lemma and invariance principle for systems with multiple hysteresis non-linearities. Int. J. of Control, 74(11):1140-1157, 2001.

[11] P.G. Park, D. Banjerdpongchai, and T. Kailath. The asymptotic stability of nonlinear (Lur'e) systems with multiple slope restrictions. IEEE Transactions on Automatic Control, 43(7):979-982, 1998.

[12] S. Tarbouriech, G. Garcia, J.M. Gomes da Silva Jr., and I. Queinnec. Stability and stabilization of linear systems with saturating actuators. Springer, London, 2011.

[13] S. Tarbouriech, I. Queinnec, and C. Prieur. Stability analysis for systems with saturation and backlash in the loop. In IEEE Conference on Decision and Control, Florence, Italy, December 2013.

[14] S. Tarbouriech, I. Queinnec, and C. Prieur. Stability analysis and stabilization of systems with input backlash. IEEE Transactions on Automatic Control, 59(2):488-494, February 2014.

[15] S. Tarbouriech, I. Queinnec, and C. Prieur. Nonstandard use of antiwindup loop for systems with input backlash. IFAC Journal of Systems and Control, 6:33-42, 2018

[16] A. Taware and G. Tao. Control of sandwich nonlinear systems. Lecture Notes in Control and Information Sciences, vol.288, Springer-Verlag, Berlin, 2003

[17] M. Turner and S. Tarbouriech. Anti-windup compensation for systems with sensor saturation: a study of architecture and structure. International Journal of Control, 82(7):1253-1266, July 2009.

[18] L. Zaccarian and A.R. Teel. Modern anti-windup synthesis. Princeton University Press, Princeton, 2011. 